\title{
A Rare Cause of Rapidly Progressive Fournier's Gangrene: Staphylococcus lungdunensis
}

\author{
Serkan Akan, Ahmet Urkmez, Ozgur Haki Yuksel, Caglar Yildirim, Ayhan Verit \\ Fatih Sultan Mehmet Research E Training Hospital, Dept. of Urology, Istanbul, Turkey
}

\begin{abstract}
Fournier's gangrene is a polymicrobial infection of perineal and scrotal regions that manifests as a rapidly progressive necrotizing fasciitis. Because of the importance of targeted antibiotic therapy, awareness of the spectrum of microbes that might be associated with lesion is imperative. Here we report an extremely rare but rapidly progressive case of necrotizing fasciitis associated with the Staphylococcus lungdunensis. J Microbiol Infect Dis 2016; 6(3): 132-135
\end{abstract}

Key words: Fournier's gangrene; Staphylococcus lungdunensis; radical debridement; broad spectrum antibiotics

\section{INTRODUCTION}

Fournier's gangrene (FG) is a polymicrobial infection of perineal and scrotal regions that manifests as a rapidly progressing necrotizing fasciitis [1]. Firstly, in 1883, it was described by Jean Alfred Fournier. In 1883 and 1884, Fournier presented five cases of a rapidly progressing gangrenous infection in five otherwise healthy young men. In the following decades different terms were used to describe this clinical entity although Wilson introduced the term 'necrotizing fasciitis' in 1952, which is still used today. In the literature numerous infectious agents including group A Streptococci, Clostridium perfringens, Staphylococcus aureus, Enterobacteriaceae, E. coli, Pseudomonas spp have been reported, and in the recent literature data related to variations in bacterial species, development of bacterial resistance, and increase in virulence have attracted much attention. Here we report an extremely rare and rapidly progressed case of necrotizing fasciitis associated with Staphylococcus lungdunensis.

\section{Case Report}

A 44-year-old male patient presented to our clinic with sudden onset of complaints of testicular swelling 24 hours previously. He did not mention any systemic disease, previous operation or chronic drug use. In patient's medical history, there was no significant feature except a self-administrated rectal enema because patient has had complaints of constipation for the last 4 days. Respiratory, cardiac, and abdominal examinations did not reveal any abnormal finding. On examination of external genitalia, scrotum was hyperemic, edematous and painful on palpitation. On the right hemiscrotum, only necrotic skin area with dimensions of $7 \times 4 \mathrm{~mm}$ was detected. Scrotal ultrasonogram was evaluated as normo-echogenic testes, and normal arteriovenous blood flow. Laboratory findings disclosed increases in the levels of inflammation markers. [WBCs $=21.4$ $\times 10^{3}, 84.4 \%$ neutrophils; glucose: $113 \mathrm{~g} / \mathrm{dL}$; BUN $13 \mathrm{mg} / \mathrm{dL}$, creatinine $0.7 \mathrm{mg} / \mathrm{dL}$, C-reactive protein: $23.7 \mathrm{mg} / \mathrm{L}]$

During observation period a remarkably rapid progression in the lesion was seen. Within onehour, scrotal skin area of nearly $40 \times 30 \mathrm{~mm}$ underwent necrosis, and purulent discharge was drained from this area. Skin discoloration, and increased skin temperature were observed along the inguinal fascia arcuate line. The patient was consulted with the department of infectious diseases, and samples were obtained for blood, urine, and lesion aspirate cultures. Meropenem $(3 \times 2 g r 24 / h)+$ vancomycin (2x1gr 24/h) treatment was initiated. Urgently, informed consent of the patient was obtained, and an extensive surgical debridement encompassing the scrotal region was performed (Figure 1). 


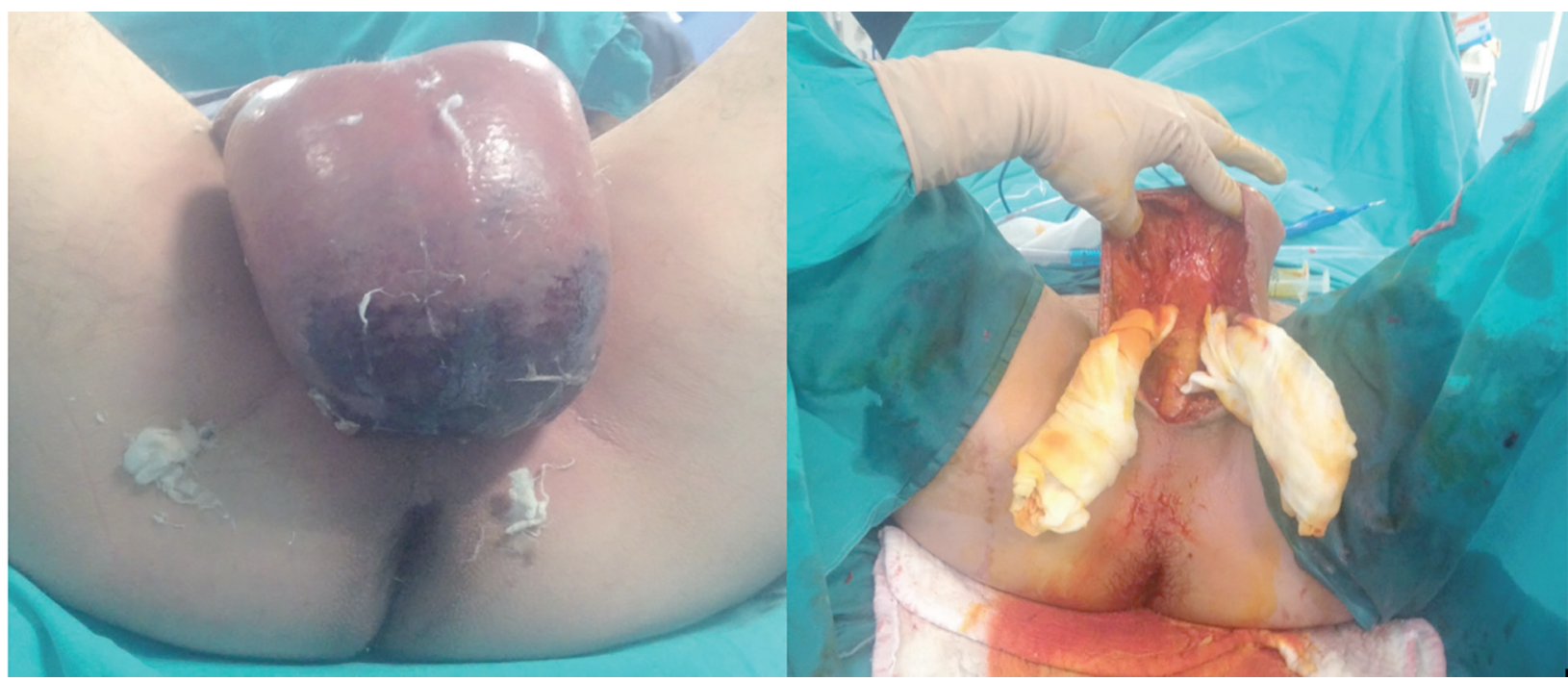

Figure 1. Gangrenous penoscrotal and inguinal tissue before and after debridement.

Bacterial growth was not detected on his urine, and blood cultures. Blood tests for HIV 1, HIV 2, hepatitis $B$, and hepatitis C, and QuantiFERON gold tuberculosis test were negative. Numerous white blood cells and gram-positive cocci were seen during microscopic examination of preoperatively obtained culture materials of aspirate, and debrided tissue samples. Final bacterial susceptibility test results revealed tetracycline, and penicillin resistant, but vancomycin susceptible staphylococcus lungdunensis. Microscopic analysis of the debrided specimen revealed areas of necrosis and acute and chronic inflammation, consistent with the diagnosis of necrotizing fasciitis.

Surgical debridement was repeated on the third, and seventh days of the hospitalization. His antibiotherapy was completed up to fourteen days. On the $15^{\text {th }}$ day, bilateral interposition flap was applied, and a Hemovac drain was placed through the surgical incision by a surgical team including urologists, and plastic, and reconstructive surgeons. The patient was discharged on the $21^{\text {st }}$ postoperative day following surgical cure (Figure 2).
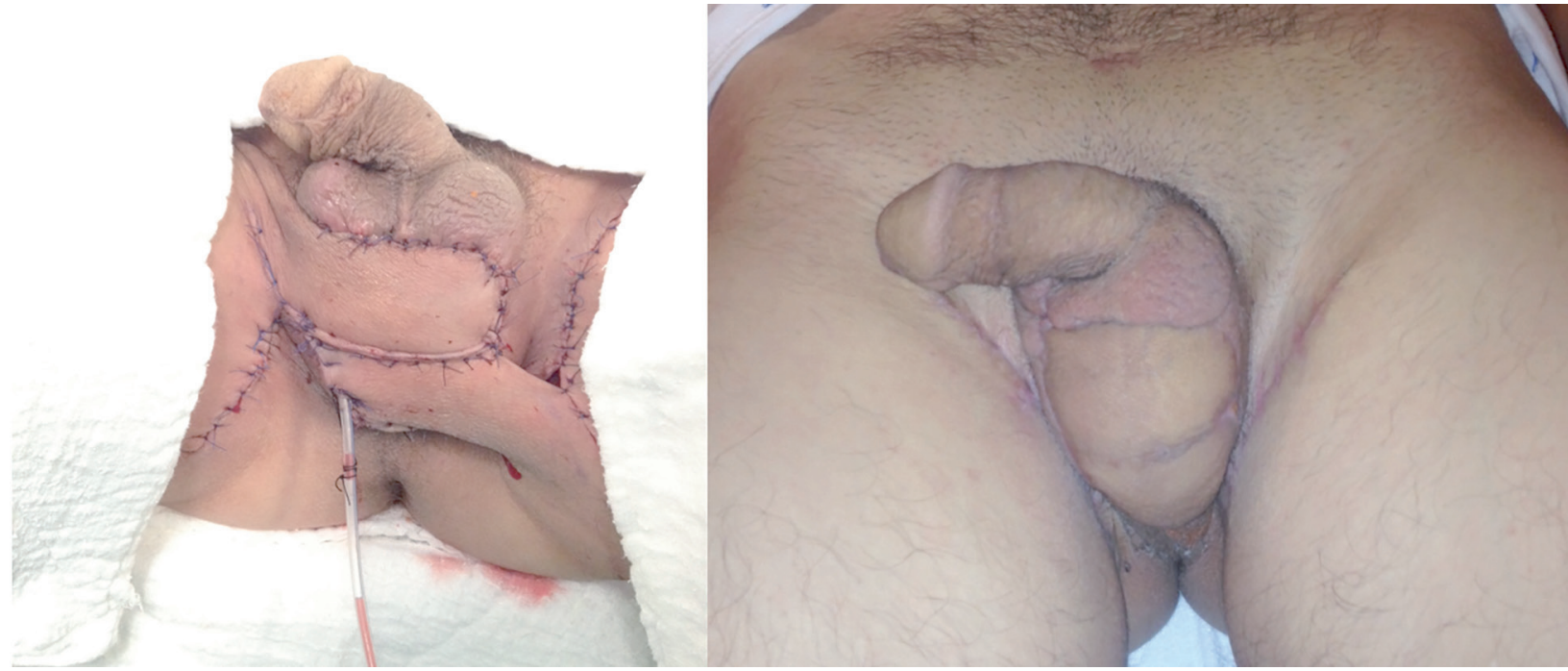

Figure 2. Bilateral interposition flap and surgical site after reconstructive surgery. 


\section{DISCUSSION}

Fournier's gangrene is a well-known, often fatal fasciitis of the pelvic floor with infectious etiology. Its overall incidence is $1 / 7500$ in the US. It is seen 10 times more frequently in men. Most often it is seen in the age bracket of 30-39 years. It develops most frequently (40\%) secondary to idiopathic causes, followed by urogenital infections, and traumas $(24 \%)$, infections of the anal region $(20 \%)$, intra-abdominal infections (10\%), gastrointestinal cancers, and use of dwelling urethral catheters. The infection commonly starts as cellulitis adjacent to the portal of entry. Early on, the involved area is swollen, erythematous, and tender as the infection begins to involve the deep fascia. Pain is prominent, and fever, Alterations in mental status, tachypnea, tachycardia, and systemic toxicity are marked [2]. The mortality rate averages approximately 20\% but ranges from $7 \%$ to $75 \%$ [3]. Predisposing factors include diabetes because of microangiopathy and polyneuropathy, alcoholism, immune-defects, and consumptive diseases [4]. Patients frequently have a history of recent perineal trauma, instrumentation, urethral stricture associated with sexually transmitted disease, or urethral cutaneous fistula. If the abdominal wall becomes involved in an obese patient with diabetes, fasciitis can spread as far as the buttocks posteriorly and the clavicle superiorly. Our patient was 44 year old and had no predisposing factors such as; diabetes, alcoholism, cancers, chronic viral diseases and tuberculosis. But the selfadministrated rectal enema may be considered as a cause of perineal trauma. Because, these phosphate content enemas increase the proportion of $\mathrm{CO}_{2}$ in the rectum and so, they raise the rectal pressure and may be a predisposing factor in the formation of Fournier's gangrene. Fournier's gangrene begins as a local infection that is caused by bacteria inhabiting the lower gastrointestinal tract, urogenital tract or the perineum. The synergistic action of aerobic and anaerobic organisms plays a major role in the progressive course of the infection [5]. While surgical debridement is the essential treatment of FG, addition of broad-spectrum antibiotics at the time of presentation and further appropriate antimicrobial therapy targeted for specific causative microbes may modify the clinical picture and are also critical in the management of this infectious process [6].

Generally necrotizing fasciitis is conventionally analyzed in two categories from microbiological aspect. Type 1 necrotizing fasciitis is character- ized by polymicrobial, synergistic infections that are caused by non-Group A streptococci, aerobic and anaerobic organisms. Type 2 necrotizing fasciitis is caused by Group A Streptococci (GAS) with or without a coexisting staphylococcal infection. These two types are considered as surgical emergencies, and targeted antibiotherapy is used [7]. Up to now various infectious agents including Group A Streptococci, Clostridium perfringens, Staphylococcus aureus, Enterobacteriaceae, E. coli, Pseudomonas spp have been reported.

Coagulase negative staphylococci (CNS) are considered as normal flora of the skin, and they are benign saprophytic agents in the body of the host. However in cases with skin trauma, and as an outcome of invasive procedures including intravenous catheter use, creation of shunt systems, and presence of artificial heart valves, and articular prostheses, pathogenic agents proliferate, and infection develops dependent on the adherence capabilities of pathogens to these foreign substances, and their abilities to escape from the tissue-protective effects of the immune system [8]. Among CNSs, S. epidermidis is the mostly isolated infectious agent, and as an infectious agent $S$. lungdunensis has been reportedly isolated in $1.1-7.1 \%$ of the bacterial cultures [9]. Staphylococcus lungdunensis is a commensal human skin bacterium first described in 1988 and since reported as a cause of prolonged and recurrent skin and soft tissue infections, as well as serious infections including meningitis, ventriculo-peritoneal shunt infection, spondylodiscitis, prosthetic joint infection, catheter-related bacteremia, endocarditis, and pacemaker-related infections $[10,11]$. S. lungdunensis belongs to the group of coagulase- negative staphylococci, however it resembles to Staphylococcus aureus with respect to pathogenicity, and virulence.

S. lungdunensis typically lacks the mecA gene, which codes for penicillin binding protein (PBP) $2 a$ and induces methicillin resistance [12]. Since in only $20 \%$ of the cases beta-lactamase positivity is detected, usually infections caused by $S$. lungdunensis can be treated with penicilline, and its derivatives. In addition $S$. lungdunensis is generally susceptible to cefoxitin, vancomycin, gentamicin, and rifampicin. In less than $10 \%$ of the cases resistance against clindamycin, and fucidic acid has been reported [10].

Although clinical course of Fournier's gangrene changes dependent on the presence of multibacterial agents, and additional individual factors, it is 
generally known to be a rapidly progressive necrotizing condition. Fournier gangrene is a true urologic emergency that demands early recognition, aggressive treatment with antimicrobial agents, and surgical debridement to reduce morbidity and mortality. Larger necrotic areas require more extensive debridement of necrotic tissue. It should not be forgotten that this condition will also effect the extent (aggressivity) of future reconstructive surgery. Within the last 4 years, we observed, and managed 12 cases (incl. the present case) with Fournier's gangrene in our clinic. Our approach to this case was not different when compared with other cases. However as a remarkable finding in this case which we detailed in the presentation of the case report was rapid progression of the necrosis, and nearly 4 -fold enlargement in the hyperemic scrotal area within one hour. Primary repair was not adequate for the cure of this condition, and required more aggressive treatment with the addition of flap advancement for the sake of surgical management, and reconstruction of the wound. We think that as an etiologic agent in a surgical emergency as Fournier's gangrene, $S$. lungdunensis which increases amount of necrotic tissue within hours or even minutes, and cited in the literature only once, should be taken into consideration, and investigated in detail in future studies.

Declaration of Conflicting Interests: The authors declare that they have no conflict of interest.

Financial Disclosure: No financial support was received.

\section{REFERENCES}

1. Korkut M, Icoz G, Dayangac M, et al. Outcome analysis in patients with Fournier's gangrene: Reports of 45 cases. Dis Colon Rectum 2003; 46:649-652.

2. Paty $R$, Smith AD. Gangrene and Fournier's gangrene. Urol Clin North Am 1992; 19: 149-62.

3. Clayton MD, Fowler JE Jr, Sharifi R, Pearl RK. Causes, presentation and survival of fifty-seven patients with necrotizing fasciitis of the male genitalia. Surg Gynecol Obstet 1990; 170: 49-55.

4. Bönner $C$, Prohm P, Störkel S. Fournier gangrene as a rare complication after stapler hemorrhoidectomy. Case report and review of the literature. Chirurg 2001; 72:1464-1466.

5. Giuliano A, Lewis F, Jr, Hadley K, Blaisdell FW. Bacteriology of necrotizing fasciitis. Am J Surg 1977; 134:52-57.

6. Shimizu T, Tokuda Y. Necrotizing fasciitis. Internal Medicine 2010; 49:1051-1057.

7. Anaya DA, Dellinger EP. Necrotizing soft-tissue infection: diagnosis and management. Clin Infect Dis 2007; 44:705-710.

8. Cunha RS, Sinzato YK, Silveira VA. Comparison of methods for the identification of coagulase-negative staphylococci. Memorias Do Instituto Oswaldo Cruz 2004; 99:855-860.

9. Akpaka PE, Christian N, Bodonaik NC, Smikle MF. Epidemiology of coagulase-negative Staphylococci isolated from clinical blood specimens at the university hospital of the West Indes. W Indian Med J 2006; 55:170-173.

10. Hellbacher C, Tornqvist E, Soderquist B. Staphylococcus lungdunensis: clinical spectrum, antibiotic susceptibility, and phenotypic and genotypic patterns of 39 isolates. Clin Microbiol Infect 2006; 12:43-49.

11. Seifert $H$, Oltmanns $D$, Becker $K$, Wisplinghoff $H$, von Eiff $C$. Staphylococcus lungdunensis pacemaker-related infection. Emerg Infect Dis 2005; 11:1283-1286.

12. Ebright JR, Penugonda N, Brown W. Clinical experience with Staphylococcus lungdunensis bacteremia: a retrospective analysis. Diagnos Microbiol Infect Dis 2004; 48:17-21. 